\title{
Effects of combined ferrous sulfate administration and exposure to static magnetic field on brain oxidative stress and emotional behavior
}

\author{
M. ELFERCHICHI, K. MAAROUFI, M. SAKLY, H. ABDELMELEK \\ Université de Carthage, Faculté des Sciences de Bizerte, Laboratoire de Physiologie Intégrée, \\ Jarzouna, Tunisia
}

\begin{abstract}
A B S T R A C T
The present study was done to investigate behavioral effects and oxidative stress in iron-treated and co-exposed static magnetic field (SMF)-iron rats. Anxiety in the elevated plus- maze test, and motor skills were also assessed in the stationary beam and suspended string tests. After behavioral tests, the rats were anesthetized and their brains were removed for biochemical analysis. The co-exposure to iron and SMF induced a significant difference in elevated plus-maze test in rats. The frequency of entries and time spent in the open arms was significantly reduced $(p<0.05)$ in the iron-and SMF-exposed group compared with the group treated with iron alone and in the control group. However, no significant difference was noticed for the motor skill test between the three groups. The biochemical investigation showed that malondialdehyde level increased $(p<0.001)$ and that glutathione level and catalase enzyme activity decreased $(p<0.001)$ in brain of iron- and SMF-exposed group. The dose of iron alone used in present study, was unable to induce any effect. However, the $128 \mathrm{mT}$ SMF in the presence of iron ions in the body can induce disruption in the emotional behavior and can produce oxidative stress in brain tissue of rats.
\end{abstract}

Key words

Static magnetic Field $\bullet$ Iron $\bullet$ Oxidative stress $\bullet$ Brain $\bullet$ Anxiety $\bullet$ Motor Skills $\bullet$ Rats

\section{Introduction}

Humans are continuously exposed to electric and/ or magnetic fields which raise the possibility of adverse health effects. Humans are exposed to static magnetic fields (SMF) resulting from the use of DC current in battery powered appliances, electric railway systems, etc. SMF are also found in occupational settings involving electrolytic processes (aluminium industry), arc-welding and more recently magnetic resonance imaging (MRI) for medical diagnosis and biomedical research.

Weak SMF may interact with biological system. In 1997, the World Health Organization and the International Commission on Non-Ionizing Radiation Protection considered that the SMF can produce various biological effects (Repacholi and Greenebaum, 1999). SMF can stimulate the biosynthesis of plasma corticosterone and the activity of metallothionein and can enhance apoptosis in female rats; this apoptosis induced by SMF could be linked to oxidative stress (Chater et al., 2005; 2006). SMF influences the kinetics of reactions in living organism with radical-pair intermediate (Grissom, 1995). Animal studies showed variable effects on behavior. Indeed, SMF exposure can affect nervous system and behavior of animal (Abdelmelek et al., 2006; Kholodov and Lebedeva, 1992), increase locomotor activity, suppress elaborated labyrinth behavior (Kholodov, 1971), and alter the cognitive functions in both human and animal. Low intensity exposures produced no effect on retention 
of an avoidance task (1.5 T for $72 \mathrm{~h}$ ) (Davis et al., 1984), locomotor activity (0.49 $\mathrm{T}$ for $2 \mathrm{~h}$ /day for 20 days) (Trzeciak et al., 1993), but affected spatial discrimination in a T-maze $(2 \mathrm{~T})$, (Levine et al., 1995). Some studies have revealed deficits on spatial memory due to the exposure to electromagnetic field (Lai, 1996; Lai et al., 1998), whereas other studies did not (Davis et al., 1984; Hong et al., 1988). From a neurobiological point of view, accumulation of iron in the brain may be cytotoxic, leading to behavioral disruptions (Sobotka et al., 1996). Indeed, iron overload increases the risk of Alzheimer's and Parkinson's diseases (Dexter et al., 1991) and other neurological disorders afflicting the brain and central nervous system (Fellman et al., 1998; Rosenzweig et al., 1999). In vivo exposure to 128 mT SMF was found to increase plasma transferrin and to decrease iron concentration in plasma (Elferchichi et al., 2011). The SMF increased the concentration of free radicals and some transitions metals, e.g., iron or copper, because these metals can produce oxygen-free radicals (ROS [reactive oxygen species]) by Fenton reaction or by interaction with cellular thiols (Meneghini, 1997). Some studies reported that excessive iron deposit in brain may generate cytotoxic free radical (Ben-Shachar et al., 1991). Jajet et al. (2002) reported that SMF in the presence of irons can increase the concentration of oxygen-free radical and thus can lead to cell death. Thus, combination of SMF exposure and iron treatment, possibly involving oxidative stress (Jajet et al., 2002), may yield larger damage at cellular level and perhaps at behavioral level. Biological systems could be simultaneously exposed to many various chemical and physical agents in the natural and occupational environments.

Scassellati et al. (2004) reported that electromagnetic field might interfere with the genotoxic activity of xenobiotics. However, there has been a lack of data regarding the progress of oxidative stress following co-exposure to SMF and xenobiotics (especially heavy metals) till date.

In the present study, an experimental approach to determine the neurobehavioral effect of simultaneous exposure to iron ions and $128 \mathrm{mT} \mathrm{SMF}$ is performed, which may result in disruption of emotional behavior. The possible prooxidative proprieties of these two agents may lead to an induction of oxidative stress in brain.

\section{Materials and methods}

\section{Animals}

Adult Wistar male rats (SIPHAT, Tunis, Tunisia), weighing 100-150 g, were randomly divided into three groups: control rats $(\mathrm{n}=11)$, treatment for iron rats $(n=11)$, and consecutive exposure to iron and SMF $(n=11)$. Animals were housed in groups of 2 animals per cages at $25^{\circ} \mathrm{C}$ under a $12: 12$ light/dark cycle, with free access to food and water. Animals were cared for in compliance with the Tunisian code of practice for the Care and Use of Animals for Scientific Purposes. The experimental protocols were approved by the Faculty Ethics Committee (Faculty of Sciences of Bizerte, Tunisia).

\section{Drug treatment}

Drug: Iron sulfate hydrate (density 1.9) was purchased from Sigma, France. Thirty-three Wistar rats were assigned to one of the three groups containing 11 rats each. One group was treated with 3 $\mathrm{mg} / \mathrm{kg}$ ferrous sulfate per day (intraperitoneal [ip]) for 5 consecutive days (d1-d5). Rats were then exposed to an SMF of $128 \mathrm{mT}$ (m Tesla) $1 \mathrm{~h} /$ day for 5 other consecutive days (d6- d10). The second group was treated with $3 \mathrm{mg} / \mathrm{kg}$ ferrous sulfate per day (ip) for 5 consecutive days and was then placed under the same conditions as the first group without the exposure to the SMF for 5 other consecutive days. The $3^{\text {rd }}$ group received saline solution for 5 days and was placed under the same conditions as the first group without exposure to the SMF during 5 other consecutive days and was served as a control

\section{Static magnetic field exposure}

The exposure system has been already described in details elsewhere (Abdelmelek et al., 2006). The intensity of SMF was measured and standardized over the total floor area of the Plexiglas cage at 128 $\mathrm{mT}$. The exposure cage was $20 \mathrm{~cm}$ long, $10 \mathrm{~cm}$ wide and $20 \mathrm{~cm}$ high. The two bobbins of the Lake Shore System were separated by $12 \mathrm{~cm}$. Male rats were exposed to SMF for $1 \mathrm{~h} /$ day during 5 consecutive days. The cage in the Lake Shore System contained two rats for each exposure. The control rats were placed in the same conditions without the exposure to the SMF. 


\section{Behavioural testing}

\section{Emotional behaviour}

Animals were tested in the elevated plus maze according to the previously published methodologies (Roy and Chapillon, 2004). The maze was made of clear painted wood. The arms were $50 \mathrm{~cm}$ long and $10 \mathrm{~cm}$ wide, and the maze was elevated at a height of $60 \mathrm{~cm}$. The closed arms were surrounded by a $50-\mathrm{cm}$ wall, whereas the open arms had $0.5-\mathrm{cm}$ edges to maximize open arm entries (Treit et al., 1993). The test was 5 min long and began with the placement of a rat in the center of the maze, with its head facing an open arm. Time spent and numbers of entries in the different parts of the maze were recorded. Increased percentage of open arm time and open arm entries was used as a measure of reduced anxiety-like behavior (Rodgers and Johnson, 1995). In addition, closed arm entries and total activity were used as measures of motor behavior. Stretched attended postures (SAPs) were also used as potential measures for anxiety-like behavior (Rodgers and Dalvi, 1997). The maze was cleaned with a $10 \%$ alcohol solution between the placement of each animal.

\section{Motor abilities}

After the emotional behavior, motor skills were examined using a battery of tow tests.

\section{Stationary beam test}

The dynamic equilibrium was tested on a beam measuring $1 \mathrm{~m}$ in length, $2 \mathrm{~cm}$ in width, and $2 \mathrm{~cm}$ in thickness, and was divided into ten segments of $10 \mathrm{~cm}$ each. Both ends of the beam were limited by cardboards. At the beginning of the experiment, the rat was placed on the middle of the beam, its body axis being perpendicular to the long axis of the beam. During the test, the distance covered and the walking time were recorded to calculate the walking speed. The trial was stopped after 3 min or when the animal fell (Jeljeli et al., 2000).

\section{Suspended string test}

At least 5 minutes after the stationary beam test, this test was designed to evaluate the muscular strength of the animals (Lalonde et al., 1996). The rats were hung by their two forepaws in the middle of an iron wire $(50 \mathrm{~cm}$ in length and $1 \mathrm{~mm}$ in diameter); their motion was limited by the cardboards placed at both ends of the wire and in front of it. The latency before falling was measured, the maximal time being fixed to $60 \mathrm{~s}$. Each animal was subjected to three trials spaced by a 15 -min interval, when it was returned to its cage with its congeners (Jeljeli et al., 2000)

\section{Tissue biochemical analysis}

After the behavioral test, the rats were anesthetized before decapitation, their brains were dissected into small pieces and weighed, it was divided into three unequal parts randomly: one part for malondialdehyde (MDA) level, one part for glutathione (GSH) level, and one part for catalase (CAT) activity. Each parts of brain were prepared in buffer containing 10 $\mathrm{mM}$ Tris, $1 \mathrm{mM}$ ethylenediaminetetraacetic acid, and $1 \mathrm{mM}$ phenylmethylsulfonyl fluoride and were centrifuged at $4,000 \mathrm{~g}$ for $20 \mathrm{~min}$.

The MDA level was determined by a method based on the reaction with thiobarbituric acid at $90-100^{\circ} \mathrm{C}$ (Esterbauer and Cheeseman, 1990). Sample aliquots were incubated with $10 \%$ trichloroacetic acid and $0.67 \%$ thiobarbituric acid. The mixture was heated on a boiling water bath for 60 min, an equal volume of $n$-butanol was added, and the final mixture was centrifuged. The absorbance of samples was determined at $532 \mathrm{~nm}$. The results were expressed as nmol/g protein in brain tissues according to a standard graphic, which was prepared with serial dilutions of standard 1,1,3,3-tetramethoxypropane.

The CAT activity was determined according to the method of Aebi (1984) and based on the determination of the rate constant $\left(\mathrm{s}^{-1}, \mathrm{~K}\right)$ of the $\mathrm{H} 2 \mathrm{O} 2$ decomposition rate at $240 \mathrm{~nm}$. The GSH level was determined by the method of Akerboom and Sies (1981). The amount of protein in the tissues was determined using Lowry's method (Lowry et al., 1951) referring to the albumin as standard.

\section{Statistical analysis}

All statistical analyses were performed with SPSS software. All results are shown as means \pm S.E.M, differences were tested using a one-way analysis of variance followed by a Bonferroni's post-hoc test for multiple comparison between experimental groups. 


\begin{tabular}{|c|c|c|c|}
\hline Elevated plus maze & Control & Iron & Iron + SMF \\
\hline Centre time & $35.95 \pm 7.87$ & $30.14 \pm 5.04$ & $50.46 \pm 16.25$ \\
\hline Closed arms' time & $221.8 \pm 16.62$ & $223.4 \pm 21.22$ & $228.8 \pm 20.68$ \\
\hline Open arms' time (\%) & $16.96 \pm 4.44$ & $17.81 \pm 7.34$ & $9.94 \pm 3.39$ \\
\hline Number of entries in central part & $7.54 \pm 1.16$ & $5.54 \pm 0.85$ & $6.45 \pm 1.3$ \\
\hline Number of closed arms' entries & $4.9 \pm 0.83$ & $3.45 \pm 0.55$ & $1.63 \pm 0.49$ \\
\hline Number of open arms' entries (\%) & $33.35 \pm 5.36$ & $32 \pm 6.53$ & $22.46 \pm 6.82 *$ \\
\hline Total activity & $7.54 \pm 1.16$ & $5.36 \pm 0.85$ & $6.36 \pm 1.3$ \\
\hline Number of rears & $2.18 \pm 0.56$ & $1.63 \pm 0.4$ & $2.45 \pm 0.75$ \\
\hline Number of head scan & $3 \pm 0.51$ & $3.45 \pm 0.72$ & $4.18 \pm 0.88$ \\
\hline Number of SAPs & $1.9 \pm 0.68$ & $2.9 \pm 0.55$ & $1.09 \pm 0.41$ \\
\hline
\end{tabular}

\section{Results}

\section{Elevated plus maze}

As shown in Fig. 1, consecutive exposure to iron and SMF slightly reduced open arm entry ratios and the time spent in the open arms; the differences were significant $\left(\left[\mathrm{F}_{(2,30)}=6.40, \mathrm{p}<0.05\right]\right.$ and $\left[\mathrm{F}_{(2,30)}=5.28\right.$, $\mathrm{p}<0.05$ ], respectively). The post hoc analysis results showed that the coexposed SMF and iron group were significantly different from the iron group and the sham group $(\mathrm{p}<0.05)$. The percentage of open arm entries was significantly reduced in the iron- and SMF-exposed group $(\mathrm{p}<0.05)$, but there was no significant difference for the percentage of time spent into the open arms in the elevated plus maze between groups. Other data showed no difference between three groups rats for total activity in the maze, vertical activity (rears), and ethological parameters (head scan toward open arms and SAPs) (Table I).

\section{Stationary beam}

As seen in Table II, no intergroup difference was found in latencies before falling (as no rat ever fell from the beam), distance covered $\left(\mathrm{F}_{(2,30)}=0.07\right.$, $\mathrm{p}>0.05)$, and walking time $\left(\mathrm{F}_{(2,30)}=0.01, \mathrm{p}>0.05\right)$ on the stationary beam.

\section{Suspended string}

As showed in Table II, the three groups have normal latencies before falling from the suspended string $\left(\mathrm{F}_{(2,30)}=0.51, \mathrm{p}>0.05\right)$.

\section{Oxidative stress}

In this study, the consecutive exposure to iron and SMF increased the MDA level $(\mathrm{p}<0.001)$ and decreased the GSH level $(\mathrm{p}=0.001)$ and CAT enzyme activity $(\mathrm{p}<0.001)$ in the brain tissue compared with the control group (Table III). Our results showed that iron treatment does not induce a reduction in brain CAT activity and GSH level. In treated rats, we did not notice an increase of MDA level compared with control rats.

\section{Discussion}

In the present study, we report that consecutive exposure to iron ( $3 \mathrm{mg} / \mathrm{kg} / \mathrm{day}$ for 5 consecutive days) and SMF (128 mT, $1 \mathrm{~h}$ /day for 5 consecutive days) can alter the emotional behaviors in the plus maze but cannot induce alteration in motor performance in stationary beam and suspended string test. Moreover, the prooxidative properties of these two agents have led to an induction of oxidative stress in brain. The activities of CAT and GSH levels were decreased, whereas the level of MDA was increased in the brain of iron- and SMF-exposed rats. During our investigation, the motor performance on the stationary beam and the suspended string test was unaffected in the iron- and SMF-exposed group, iron group, and the sham group. The present results show that rats exposed to SMF were not impaired in any of these tests (see (Elferchichi et al., 2011), for a similar result). This result shows that the impairment of the emotional behavior in the elevated plus maze is due to specific anxiety induced by consecutive iron and SMF exposure as opposed to a generalized reduction in cerebral activation or to a loss of muscle strength. 

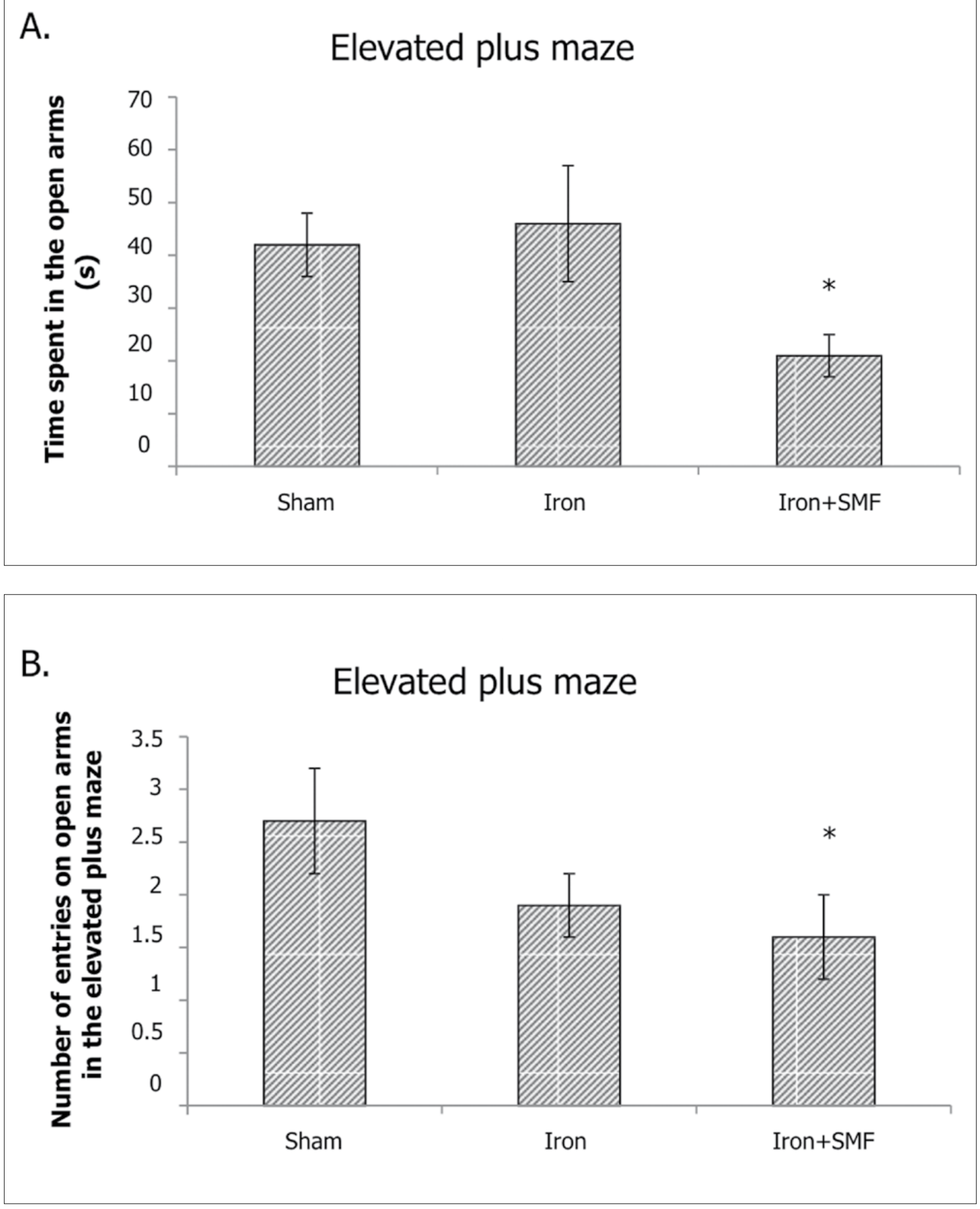

Fig. 1. - (A) Time spent in the open arms. (B) Number of entries on open arms in the elevated plus maze test for Sham $(n=11)$, iron $(n=11)$ and iron- and SMF-exposed $(n=11)$ groups. Data are given as mean \pm S. E.M. Follow-up comparisons: * $\mathrm{p}<0.05$. 


\begin{tabular}{|l|c|c|c|}
\hline \multicolumn{4}{|l|}{ Table ll.- Motor coordination and muscle strength of sham $(n=11)$, iron $(n=11)$ and iron + SMF $(n=11)$ rats (means \pm S.E.M. per trial). } \\
\hline Test & Sham & Iron & Iron + SMF \\
\hline Stationary beam & \multicolumn{3}{|l|}{} \\
\hline Latencies (s) & 180 & 180 & 180 \\
\hline Walking time (s) & $7.41 \pm 2.62$ & $7.26 \pm 2.44$ & $6.91 \pm 2.91$ \\
\hline Distance (cm) & $116.4 \pm 38.03$ & $103.6 \pm 36.75$ & $96.36 \pm 39.07$ \\
\hline Suspended string & \multicolumn{3}{|l|}{} \\
\hline Latencies (s) & $9.25 \pm 2.17$ & $9.69 \pm 1.4$ & $7.72 \pm 0.5$ \\
\hline
\end{tabular}

In conclusion, our results from the elevated plus maze test raised significant difference between sham, iron group, and iron- and SMF-exposed group. Results showed a significant decrease in the number of entries and time spent in the open arms in response to exposure to iron and SMF compared with sham and iron-exposed groups. This result shows that the exposure of rats to iron and SMF could induce anxiety in the elevated plus maze. However, SMF alone (128 mT, $1 \mathrm{~h} /$ day for 5 consecutive days) does not induce a clear effect in emotional behavior in the elevated plus maze but lead to cognitive impairments or at least to substantial attention disorders in the Morris water maze (Ammari et al., 2008). Recent study of Maaroufi et al. (2013) with similar protocol show that, combination of the two treatments did not produce any deficit in cognitive behavior. Studies on electromagnetic fields (EMF) provide some hints on the interaction between magnetic fields and iron even if it is possible that EMF and SMF do not induce similar effects. Consistent effects have been found in a recent study comparing the behavior of rats with iron treatment to rats with combined iron- $150 \mathrm{kHZ}$ EMF treatments (Maaroufi et al., 2009).

Our investigations showed that the exposure to iron alone $(3 \mathrm{~m} / \mathrm{Kg} /$ day for 5 consecutive days) does not induce significant difference in emotional behavior of rats in the elevated plus maze. In contrast, some studies proved that iron overload can induce deficit in cognitive functions and can affect behavior of rats. Maaroufi et al. (2009) show that learning abilities of iron-3.0-treated rats were affected in the Water maze. Archer and Fredriksson (2007) proved that postnatal iron overload of mice on day 10-12 postpartum at doses $7.5 \mathrm{mg} / \mathrm{kg}$ altered behaviors, hypoactivity, and later hyperactivity during the 60-min test session in adult life.

Similarly Fredriksson et al. (2000) reported that iron overload in young rats induced deficit in memory. It is known that the postnatal period is critical for establishment of normal iron in adult brain and that the excess of iron in brain during the critical neonatal period can generate cytotoxic free radical in adult life (Ben-Shachar et al., 1991) and can lead to cognitive impairment (Fredriksson et al., 1999; de Lima et al., 2005).

Some studies have shown that the association between iron and SMF can produce ROS. Jajte et al. (2002) reported that SMF in the presence of iron can increase the concentration of ROS and can thus lead to cell death. In our study, iron and SMF may induce perturbations in the brain and may alter the emotional behavior. A previous study suggested that extremely low frequency (ELF) as electric fields can affect the nervous system and may have a specific effect on the risk of brain tumor (Kheifets et al., 1995). Change in blood-brain barrier morphology, electrophysiology, neurotransmitter functions, cellular metabolism, calcium efflux, and genetic effects have been reported in the brain of animals after exposure to ELF (Guenel, 1997).

Previously, it has been demonstrated that exposure to low electromagnetic field cause DNA damage in the brain cells of rats with the involvement of ROS (Singh and Lai, 1998). Also, Zmyslony et al. (2000) showed that exposure to $7 \mathrm{mT}$ SMF can induce DNA damage in rat lymphocytes if the cells were simultaneously treated with FeCl2. Similarly, exposure to $7 \mathrm{mT}$ SMF in the presence of iron ions can increase the concentration of ROS and can thus lead to apoptosis and necrosis of rat lymphocytes (Jajte et al., 2002). In another study, exposure to $5 \mathrm{mT} \mathrm{SMF}$ under iron ions stimulation increased lipid peroxidation in isolated rat liver microsomes (Zmyslony and Jajte, 1998).

In our study, the consecutive exposure to iron and SMF can induce anxiety in rats. We consider that SMF exposure increases the effect of iron overload in the brain because SMF may affect the permeability 
Table III. - Brain tissue MDA, GSH, and CAT enzyme activity of sham, iron, and SMF- and Iron-exposed groups.

\begin{tabular}{|c|c|c|c|}
\hline Parameters & Sham & Iron & Iron + SMF \\
\hline MDA (nmol/g prot) & $102.82 \pm 2.26$ & $107.95 \pm 1.94$ & $126.15 \pm 1.84 a, b$ \\
\hline GSH ( $\mu \mathrm{mol} / g$ prot) & $1.52 \pm 0.06$ & $1.33 \pm 0.08$ & $1.06 \pm 0.06^{b}$ \\
\hline CAT (K/g prot) & $1.23 \pm 0.05$ & $1.15 \pm 0.03$ & $0.89 \pm 0.04 a . b$ \\
\hline \multicolumn{4}{|c|}{$\begin{array}{l}\text { SMF (static magnetic field); MDA (malondialdehyde); GSH (glutathione); CAT (catalase). Values are expressed as mean } \pm \\
\text { S.E.M calculated from } n=11 \text { in each group. }\end{array}$} \\
\hline
\end{tabular}

of blood-brain barrier. The excess of iron might then penetrate in the brain and generate free radicals. The excess of iron in brain induced by SMF exposure might produce alteration of dopamine, which is an essential neurotransmitter, because dopaminergic neurons are thought to be vulnerable to oxidative stress (Barkats et al., 2002). The biochemical analysis in the brain of our three groups showed that consecutive exposure to iron and SMF induced numerous indicators of oxidative stress. This includes decrease in the levels of antioxidants such as CAT activity and GSH level and increased levels of biomarkers of lipid peroxidation such as MDA, but the iron alone at the concentration of $3 \mathrm{mg} / \mathrm{Kg} /$ day during 5 consecutive days did not induce oxidative stress in brain. The oxidative stress induced by iron and SMF in brain may be the result of increased ROS. Moreover, the increase in the concentration of ROS produced lipid peroxidation in membranes (Aristarkhov et al., 1983; Meneghini, 1997). Therefore, the level of ROS induced by iron could be additionally enhanced by the exposure to SMF as a result of the magnetic fields effect due to radical pair mechanisms. Similarly, Jajte et al. (2002) reported that exposure of lymphocytes to a $7 \mathrm{mT}$ SMF and iron ions may simulate free radical reactions involving ROS and may thus lead to cell death.

The present experiment showed that ip exposure of rats to iron $(3 \mathrm{mg} / \mathrm{kg}, \mathrm{b}, \mathrm{wt})$ during 5 days and then to SMF (128 mT) during $1 \mathrm{~h} /$ day for the next 5 consecutive days was able to alter emotional behavior of rats in elevated plus maze, but only iron supplementation did not alter the behavior of rats. It was also noticed that no significant difference was obtained in the stationary beam and suspended string test in each group. Therefore, it was suggested that $128 \mathrm{mT}$ SMF in the presence of iron ions can induce oxidative stress via the increase of oxygen-free radicals in brain.

\section{Acknowledgements}

This work was supported by the Tunisian Ministry of High Education and Scientific Research

\section{References}

Abdelmelek H., Molnar A., Servais S., Cottet-Emard J.M, Pequignot J.M., Favier R., Sakly M. Skeletal muscle HSP72 and norepinephrine response to static magnetic field in rat. J Neural Transm., 113: 821-827, 2006.

Aebi H. Catalase in vitro. Methods Enzymol., 105: 121-6, 1984.

Akerboom T.P. and Sies H. Assay of glutathione, glutathione disulfide, and glutathione mixed disulfides in biological samples. Methods Enzymol., 77: 373-82, 1981.

Ammari M., Maaroufi K., Jeljeli M., Roy V., Sakly M. Abdelmelek H. Static magnetic field exposure affect behaviour and learning in rats. Electromagn. Biol. Med., 27: 185-196, 2008.

Archer T. and Fredriksson A. Functional consequences of iron overload in catecholaminergic interactions: the youdim factor. Neurochem. Res., 32: 1625-39, 2007.

Aristarkhov V.M., Sineva I.V., Merkulova L.M. Effect of the magnetic field on the changes in heart rate and serotonin content of isolated frog heart. Izv. Akad. Nauk. SSSR Biol., 1: 111-21, 1983.

Barkats M., Millecamps S., Bilang-Bleuel A., Mallet J. Neuronal transfer of the human $\mathrm{Cu} / \mathrm{Zn}$ superoxide dismutase gene increases the resistance of dopaminergic neurons to 6- hydroxydopamine. $J$ Neurochem., 82: 101-9, 2002.

Ben-Shachar D., Riederer P., Youdim M.B. Ironmelanin interaction and lipid peroxidation: implications for Parkinson's disease. J. Neurochem., 57: 1609-14, 1991. 
Chater S., Abdelmelek H., Couton D., Joulin V., Sakly M., Ben Rhouma K. Sub-acute exposure to magnetic field induced apoptosis in thymus female rats. Pak. J. Med.Sci., 21: 292-297, 2005.

Chater S., Abdelmelek H., Douki T., Garrel C., Favier A., Sakly M., Ben Rhouma K. Exposure to static magnetic field of pregnant rats induces hepatic GSH elevation but not oxidative DNA damage in liver and kidney. Arch. Med. Res., 37: 941-946, 2006.

Davis H.P., Mizumori S.J., Allen H., Rosenzweig M.R., Bennett E.L., Tenforde T.S. Behavioral studies with mice exposed to DC and $60-\mathrm{Hz}$ magnetic fields, Bioelectromagnetics., 5: 147-164, 1984.

de Lima M.N., Polydoro M., Laranja D.C., Bonatto F., Bromberg E., Moreira J.C., Dal-Pizzol F., Schroder N. Recognition memory impairment and brain oxidative stress induced by postnatal iron administration. Eur. J. Neurosci., 21: 2521-2528, 2005.

Dexter D.T., Carayon A., Javoy-Agid F., Agid Y., Wells F.R., Daniel S.E., Lees A.J., Jenner P., Marsden C.D. Alterations in the levels of iron, ferritin and other trace metals in Parkinson's disease and other neurodegenerative diseases affecting the basal ganglia. Brain, 114: 1953-1975, 1991.

Elferchichi M., Ammari M., Maaroufi K., Sakly M., Abdelmelek H. Effects of exposure to static magnetic field on motor skills and iron levels in plasma and brain of rats. Brain Injury., 25: 901-8, 2011.

Esterbauer H. and Cheeseman K.H. Determination of aldehydic lipid peroxidation products: malonaldehyde and 4-hydroxynonenal. Methods Enzymol., 186: 407-21, 1990.

Fellman V., Rapola J., Pihko H., Varilo T., Raivio K.O. Iron-overload disease in infants involving fetal growth retardation, lactic acidosis, liver haemosiderosis, and aminoaciduria. Lancet., 351: 490-3, 1998.

Fredriksson A, Schroder N., Eriksson P., Izquierdo I., Archer T. Maze learning and motor activity deficits in adult mice induced by iron exposure during a critical postnatal period. Brain Res. Dev. Brain. Res., 119: 65-74, 2000.

Fredriksson A., Schroder N., Eriksson P., Izquierdo I., Archer T. Neonatal iron exposure induces neurobehavioural dysfunctions in adult mice. Toxicol. Appl. Pharmacol., 159: 25-30, 1999.

Grissom C.B. Magnetic field effects in biology: a survey of possible mechanisms with emphasis on radicalpair recombination. Chem. Rev., 95: 3-24, 1995.

Guenel P. 50-60 Hz electromagnetic fields and cancer risk. Rev. Epidemiol. Sante Publique, 45: 93-5, 1997.
Hong C.Z., Huestis P., Thompson R., Yu J. Learning ability of young rats is unaffected by repeated exposure to a static electromagnetic field in early life. Bioelectromagnetics, 9: 269-273, 1988.

Jajte J., Grzegorczyk J., Zmyslony M., Rajkowska E. Effect of $7 \mathrm{mT}$ static magnetic field and iron ions on rat lymphocytes: apoptosis, necrosis and free radical processes. Bioelectrochemistry, 57: 107111, 2002.

Jeljeli M., Strazielle C., Caston J., Lalonde R. Effects of centrolateral or medial thalamic lesions on motor coordination and spatial orientation in rats. Neurosci. Res., 38: 155- 164, 2000.

Kheifets L.I., Afifi A.A., Buffler P.A., Zhang Z.W. Occupational electric and magnetic field exposure and brain cancer: a meta-analysis. J. Occup. Environ. Med., 37: 1327-41, 1995.

Kholodov Yu A. and Lebedeva N. N. Reaction of Human Nervous System to Electromagnetic Fields. Nauka, Moscow, 1992.

Kholodov Yu. A. Influence of Magnetic Fields on Biological Objects. JPRS \#63038, National Technical Information Service, U. S. Department of Commerce, 1971.

Lai H. Spatial learning deficit in the rat after exposure to a $60 \mathrm{~Hz}$ magnetic field, Bioelectromagnetics., 17: 494-496, 1996.

Lai H., Carino M.A., Ushijima I. Acute exposure to a $60 \mathrm{~Hz}$ magnetic field affects rats' water- maze performance. Bioelectromagnetics., 19: 117-122, 1998.

Lalonde R., Filali M., Bensoula A.N., Lestienne F. Sensorimotor learning in three cerebellar mutant mice. Neurobiol. Learn. Mem., 65: 113-20, 1996.

Levine R., Dooley J., Bluni T. Magnetic field effects on spatial discrimination and melatonin levels in mice. Physiol. Behav., 58: 535-537, 1995.

Lowry O.H., Rosebrough N.J, Farr A.L., Randall R.J. Protein measurement with the Folin phenol reagent. J. Biol. Chem., 193: 265-75, 1951.

Maaroufi K., Had-Aissouni L., Melon C., Sakly M., Abdelmelek H., Poucet B., Save E. Effects of prolonged iron overload and low frequency electromagnetic exposure on spatial learning and memory in the rat. Neurobiol Learn Mem., 92, 345-55, 2009.

Meneghini R. Iron homeostasis, oxidative stress, and DNA damage. Free Radic. Biol. Med., 23: 783792, 1997.

Repacholi M.H. and Greenebaum B. Interaction of static and extremely low frequency electric and magnetic fields with living systems: health effects and research needs, Bioelectromagnetics., 20: 133-160, 1999. 
Rodgers R.J. and Dalvi A. Anxiety, defence and the elevated plus-maze. Neurosci. Biobehav. Rev., 21: 801-810, 1997.

Rodgers R.J., Johnson N.J. Factor analysis of spatiotemporal and ethological measures in the murine elevated plus-maze test of anxiety. Pharmacol. Biochem. Behav., 52: 297-303, 1995.

Rosenzweig P.H. and Volpe S.L. Iron thermoregulation and metabolic rate. Crit. Rev. Food Sci. Nutr., 39: 131-48, 1999.

Roy V., Chapillon P. Further evidences that risk assessment and object exploration behaviours are useful to evaluate emotional reactivity in rodents. Behav. Brain. Res., 154: 439-48, 2004.

Scassellati Sforzolini G., Moretti M., Villarini M., Fatigoni C., Pasquini R. Evaluation of genotoxic and/or co-genotoxic effects in cells exposed in vitro to extremely-low frequency electromagnetic fields. Ann. Ig., 16: 321-40, 2004.

Singh N. and Lai H. $60 \mathrm{~Hz}$ magnetic field exposure induces DNA crosslinks in rat brain cells. Mutat. Res., 400: 313-20, 1998.
Sobotka T.J., Whittaker P., Sobotka J.M., Brodie R.E., Quander D.Y., Robl M., Bryant M., Barton C.N. Neurobehavioral dysfunctions associated with dietary iron overload. Physiol Behav., 59: 213-9, 1996.

Treit D., Menard J., Royan C. Anxiogenic stimuli in the elevated plus-maze. Pharmacol. Biochem. Behav., 44: 463-469, 1993.

Trzeciak H., Grzesik J., Bortel M., Kuska R., Duda D., Michnik J., Małecki A. Behavioral effects of long-term exposure to magnetic fields in rats. Bioelectromagnetics., 14: 287- 97,1993.

Zmyslony M. and Jajte J.M. The role of free radicals in mechanisms of biological function exposed to weak, constant and net magnetic fields. Med. Pr., 49: 177-186, 1998.

Zmyslony M., Palus J., Jajte J., Dziubaltowska E., Rajkowska E. DNA damage in rat lymphocytes treated in vitro with iron cations and exposed to 7 mT magnetic fields (static or $50 \mathrm{~Hz}$ ). Mutat. Res., 453: 89-96, 2000. 